\title{
Characterization of a developmentally regulated amino acid transporter (AAT1p) of the rust fungus Uromyces fabae
}

\author{
CHRISTINE STRUCK', MICHAEL ERNST ${ }^{1}$ AND MATTHIAS HAHN $2, *$ \\ 'Fachbereich Biologie, Universität Konstanz, 78457 Konstanz, Germany, \\ ${ }^{2}$ Fachbereich Biologie, Universität Kaiserslautern, 67653 Kaiserslautern, Germany
}

\begin{abstract}
SUMMARY
In the rust fungus Uromyces fabae, invasion of the host plant and haustorium formation are accompanied by the activation of many genes ( $P / G S=$ in planta induced genes). In addition to the previously described $A A T 2$ (PIG2), AAT1 (PIG27) was found to encode a protein with a high similarity to fungal amino acid permeases. AAT1 transcripts are present in germinated hyphae and throughout the mycelium later in the infection process, but occur at the highest levels in haustoria. Expression of AAT1p in a histidine uptake-defective yeast mutant revealed energy-dependent transport of ${ }^{14} \mathrm{C}$-histidine, with a $K_{\mathrm{M}}$ value of $25.8 \mu \mathrm{M}$. In addition, complementation analysis revealed AAT1-dependent transport for lysine. Using Xenopus oocytes as expression system, AAT1pdependent symport of protons with a broad spectrum of amino acids was observed, with the highest activities obtained with histidine and lysine. These results confirm that in rust fungi, the expression of amino acid transporters is developmentally regulated and occurs preferentially in the parasitic phase of development.
\end{abstract}

\section{INTRODUCTION}

Plant pathogenic rust fungi are highly adapted to an obligate biotrophic life style. Germinated rust spores invade the host tissue by forming a series of elaborate infection structures. Within the host tissue, the mycelium grows by means of intercellular hyphae and haustoria that penetrate into living host cells (Hahn, 2000). Both the extensive net of intercellular hyphae and haustoria represent potential sites for substrate uptake. However, haustoria seem to be particularly suited for providing nutrient supply to the fungus, because of their close contact to the cytoplasm of mesophyll cells. Although axenic cultures have been established for a number of rust species, indicating that no special nutrients are required for growth (for review see Staples, 2000), little is known about the

‘Correspondence: E-mail: address: hahn@rhrk.uni-kl.de nutritional requirements for their development and reproduction in living host plants. Previous studies with Puccinia graminis have provided indirect evidence that various amino acids are taken up from the host plant (Jäger and Reisener, 1969; Mendgen, 1981).

In recent years, increasing molecular evidence has been obtained for the long-suspected role of rust haustoria in nutrient acquisition, and the involvement of a plasma membrane $\mathrm{H}^{+}$ATPase and proton-driven amino acid and sugar transporters (Mendgen et al., 2000). The activity of the plasma membrane ATPase was found to be considerably higher in haustoria than in spores and germlings of the fungus (Struck et al., 1996). Subsequent cloning and expression of the corresponding gene ( $U \mathrm{f}$ PMA1) indicated a complex pattern of regulation, both on transcriptional and post-transcriptional levels (Struck et al., 1998). Differential screening of a haustorium-specific CDNA library resulted in the isolation of a large number of rust genes, showing preferential expression in parasitically growing hyphae and haustoria (Hahn and Mendgen, 1997). Two of these genes (PIGZ and PIG27, renamed AAT2 and AAT1; Mendgen et al., 2000), showed considerable similarity to amino acid transporters of other organisms, including yeast. Using RNA blot hybridization and immunofluorescence microscopy, the expression of AAT2p (PIG2p) was detected only in haustoria, and not in early infection structures or in intercellular hyphae (Hahn et al., 1997).

In this study we have performed a structural and functional analysis of the $A A T 1$ gene. We observed that, while $A A T 1$ is structurally similar to $A A T 2$, its regulation is different. By using yeast and Xenopus oocytes as heterologous hosts, we have characterized the kinetic features and amino acid specificity of the AAT1p transporter.

\section{RESULTS}

\section{Structural analysis of $A A T 1$}

The original AAT1 (PIG27) clone from the haustorium-specific Uromyces fabae $\lambda$ gt 10 CDNA library carried a 997 bp insert that contained only part of the coding region for a putative amino transporter. This AATI CDNA fragment was used to screen a 
$\lambda E M B L 3$ genomic DNA library of $U$. fabae. Two clones were isolated, with inserts of 15 and $18 \mathrm{~kb}$, respectively, each containing an overlapping region of at least $8 \mathrm{~kb}$ as determined by comigrating EcoRI fragments and by PCR analysis using combinations of AAT1-specific and $\lambda E M B L 3$-specific primers. Partial sequencing revealed that both genomic clones carried the same gene (not shown). One of the genomic $A A T 1$ clones was chosen for sequencing of the complete $A A T 1$ gene. The sequenced region covered $4694 \mathrm{bp}$, including $1009 \mathrm{bp}$ upstream of the putative start codon, 3108 bp of coding region plus introns, and 577 bp of the $3^{\prime}$ downstream region (accession no. AJ308252). Within this sequence, the coding region including the putative ATG start codon was identified by alignment of the deduced amino acid sequence with the AAT2 (PIG2)-encoded protein (Hahn et al., 1997). Using RT-PCR with mRNA from $U$. fabae haustoria and the appropriate primers (see Experimental procedures), the AAT1 CDNA covering the coding region was isolated. The longest open reading frame starting with ATG resulted in a predicted protein (AAT1p) with 552 amino acids and a molecular mass of $60.6 \mathrm{kDa}$, similar to other amino acid permeases from fungi.

Multiple sequence alignments of several known amino acid transporters from a variety of organisms suggested that AAT1p belongs to the yeast amino acid transporter family (YAT, TC2.A.3.10; Jack et al., 2000) (Fig. 1). Similarities were also

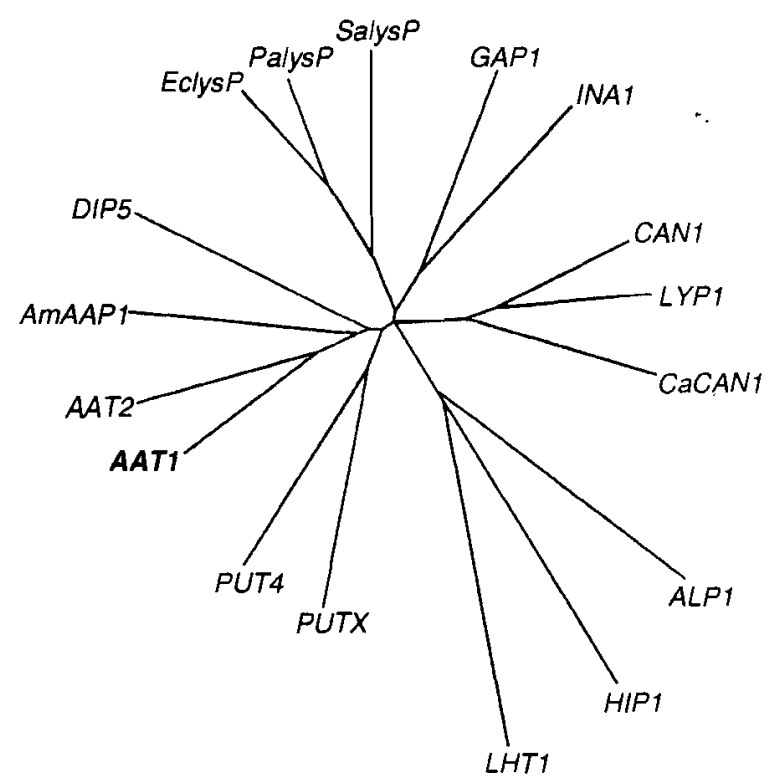

Fig. 1 Phylogenetic comparison of AAT1p with different amino acid transporters. Accession numbers: Uromyces fabae AAT 1p: AJ308252; AAT2p: U81794; Amanita muscaria AmAAP1: AJ223504; Saccharomyces cerevisiae DIP5: P53388, GAP1:P19145, CAN1: P04817, LYP1: P32487, ALP1: P38971, HIP 1: P06775, PUT4: P15380; Candida albicans CaCAN1: P43059; Emericella nidulans PUTX: S42708; Trichoderma harzianum INA1: P34054; Arabidopsis thaliana I.HT1: U39782; Escherichia coli EclysP: P25737; Pseudomonas aeruginosa PalysP: G08016: Staphylococcus aureus SalysP: BAB42772.

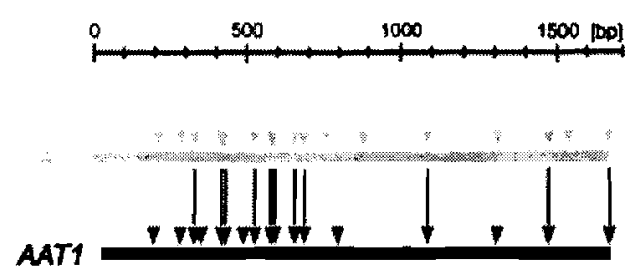

Fig. 2 Similar positions of introns in the AAT1 and $A A T 2$ genes of Uromyces fabae. The coding regions of both genes are shown, and the introns marked by arrowheads. Vertical lines indicate intron pairs located at the same positions.

found to bacterial amino acid transporters (members of the AAT family, according to lack et al., 2000) and to the LHT1 encoded transporter from Arabidopsis thaliana. However, AAT1p shares the highest similarity with another putative amino acid permease of $U$. fabae, AAT2p (55.1\% identity; Hahn et al., 1997). Both are closely related to the general amino acid permease (AmAAT1p) of another basidiomycete, the ectomycorrhizal fungus Amanita muscaria (AAT1p to AmAAT1p: $45.9 \%$ identity; Nehls et al., 1999). Comparison of the genomic and the PCR-amplified CDNA sequences of $A A T 1$ revealed the presence of 17 introns, which is the same number of introns as found in AAT2 (Hahn et al., 1997). When the intron positions within the coding regions of $A A T 1$ and $A A T 2$ were analysed, it was found that 11 introns are located at conserved positions in both genes (Fig. 2).

\section{Developmental expression of AAT1}

The expression of $A A T I$ during rust development was analysed by Northern hybridization analysis. Whereas no transcript was detected in resting uredospores, significant levels of $A A T 1$ mRNAs were found in germinated spores and infection structures formed in vitro (Fig. 3). The highest mRNA levels were observed in haustoria, and in rust-infected leaves which contain fungal mycelium including haustoria. Two different size classes ( 2.3 and $2.5 \mathrm{~kb}$ ) of AAT1 transcripts were evident; whereas smaller transcripts of about $2.3 \mathrm{~kb}$ dominated in germinated spores and in $6 \mathrm{~h}$-old infection structures (predominantly appressoria), AAT1 mRNAs of about $2.5 \mathrm{~kb}$ were increasingly prevalent in infection structures which had developed for 12, 18 and $24 \mathrm{~h}$ (which had successively formed substomatal vesicles, infection hyphae and haustorial mother cells (Deising et al., 1991)). In the parasitic mycelium (haustoria and rust infected leaves), only the larger transcripts were clearly detected (Fig. 3). Using RT-PCR with RNA obtained from germinating spores $(2.3 \mathrm{~kb}$ transcript predominating) and from haustoria ( $2.5 \mathrm{~kb}$ transcript predominating), we found that the sequences covering the $A A T I$ coding region were indistinguishable from each other (data not shown). Therefore, the two size classes of transcripts are likely to be the products of the same gene. 


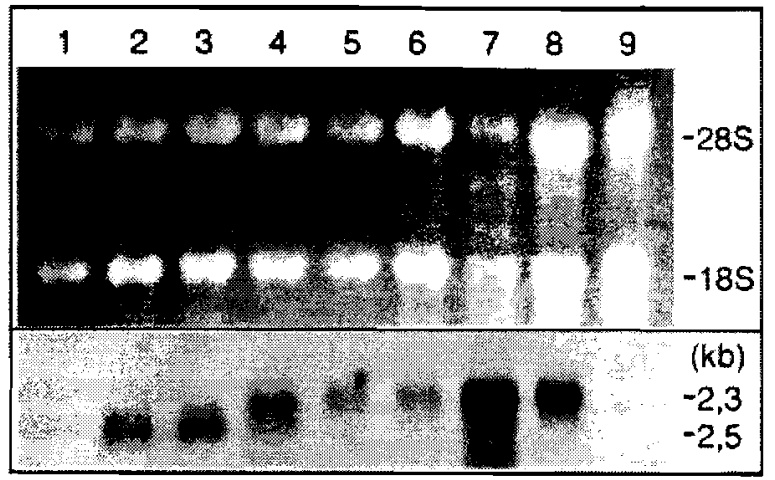

Fig. 3 Expression of $A A T /$ transcripts during different stages of rust development. The upper part shows the nylon membrane containing ethidium bromide stained total RNA after Northern blotting. The bands corresponding to 285 and 185 RNAs are indicated. The lower part shows the corresponding bands hybridizing to AAT1. (Lane 1) Dormant uredospores; (2) $4 \mathrm{~h}$ germinated spores; $(3-6)$ In vitro differentiated infection structures grown for the following times: (3) 6 h, (4) 12 h, (5) 18 h, (6) 24 h; (7) Isolated haustoria; (8) Rust-infected Vicia faba leaves, 5 d.p.i.: (9) Non-infected leaves.

\section{Copy number and occurrence of AATI and $A A T 2$ in other fungi}

Genomic Southern hybridization analysis indicated the presence of a single copy of $A A T 1$ in the $U$. fabae genome (data not shown). To test whether AAT1-like genes are also present in other fungi, heterologous genomic Southern blotting assays-were performed. Using reduced stringency conditions for hybridization, $A A T 1$ probes only gave significant signals with the DNA of two other rust fungi, namely Puccinia graminis f.sp. triticiand Uromyces vignae (Fig. 4). Very weak signals were obtained with DNA from the ascomycete Magnaporthe grisea, and no hybridization was observed with DNA from various other fungi (data not shown). When AAT2 DNA was used as a hybridization probe, a similar result was obtained; unambiguous hybridization signals were observed with DNA from the rusts but not from other fungi (Fig. 4; data not shown). There was no evidence of cross-hybridization between the two genes, because the size of the EcoRI fragments of $U$. fabae hybridizing to $A A T 1$ and $A A T 2$ are different. Taken together, the $A A T 1$ and $A A T 2$ orthologs seem to exist in rust fungi but not in other basidio- and ascomycetes.

\section{Functional expression of AAT1p in yeast}

To confirm the role of AAT1p as an amino acid transporter, a CDNA carrying the AAT1 coding region was amplified and cloned into the yeast expression vector pDR195. The resulting plasmid PAAT1-YE was used for transformation of the Saccharomyces cerevisiae strain JT16 which is defective in biosynthesis and uptake of histidine (Tanaka and Fink, 1985). While JT16 transformants

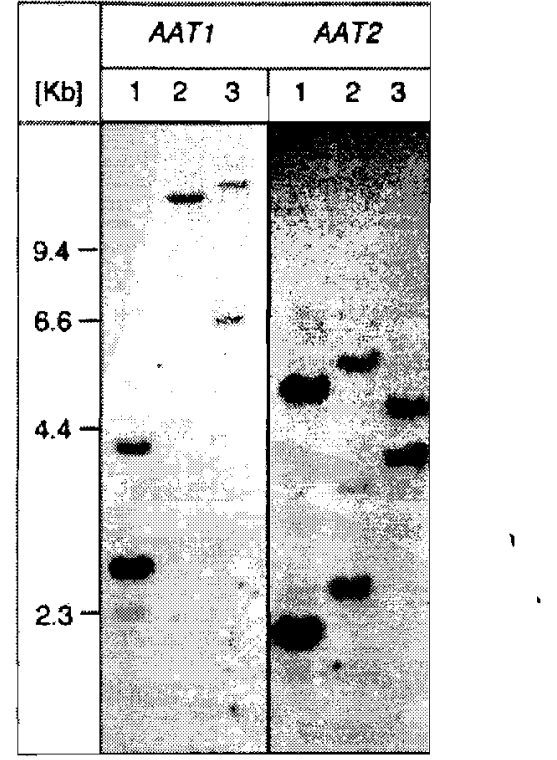

Fig. 4 Interspecies hybridization of $A A T I$ and $A A T 2$ within rust fungi. Total genomic DNAs, digested with EcoRI, from Uromyces fabae (lane 1), Uromyces vignae (lane 2) and Puccinia graminis f.sp. tritici (lane 3), were subjected to Southern blot hybridization with digoxigenin-labelled cDNA probes.

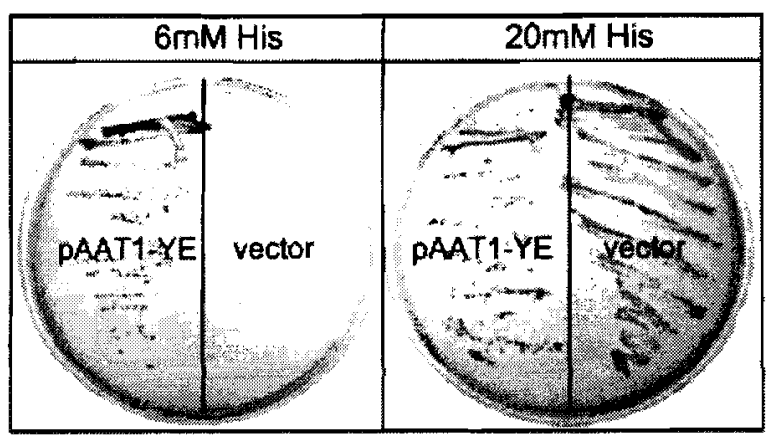

Fig. 5 Complementation of the histidine uptake defect of the yeast mutant JT 16 by the $U$. fabae gene AAT1. JT16 carrying either the expression plasmid PAAT - YE or the vector PDR195 was grown on SC medium containing 6 or $20 \mathrm{~mm}$ histidine.

carrying pDR195 were unable to grow on SC containing up to $6 \mathrm{~mm}$ histidine, transformants carrying PAAT1-YE grew well on SC medium with low histidine concentrations (Fig. 5). This result demonstrated that $A A T I$ encodes a histidine transporter.

In order to characterize the transport properties of AAT1p, $S$. cerevisiae strain JT16 (pAAT1-YE) was used in uptake studies with ${ }^{14} \mathrm{C}$-labelled histidine as a substrate. Kinetic studies indicate that AAT1p mediates a saturable, concentration-dependent uptake of histidine into the yeast JT16 (PAAT1-YE), with a putative $V_{\max }$ of $23 \mathrm{nmol} / \mathrm{mg}$ protein $/ \mathrm{min}$ and $\mathrm{a} K_{\mathrm{M}}$ of $25.8 \mu \mathrm{M}$, indicating that AAT1p is a high-affinity amino acid permease (Fig. 6). 


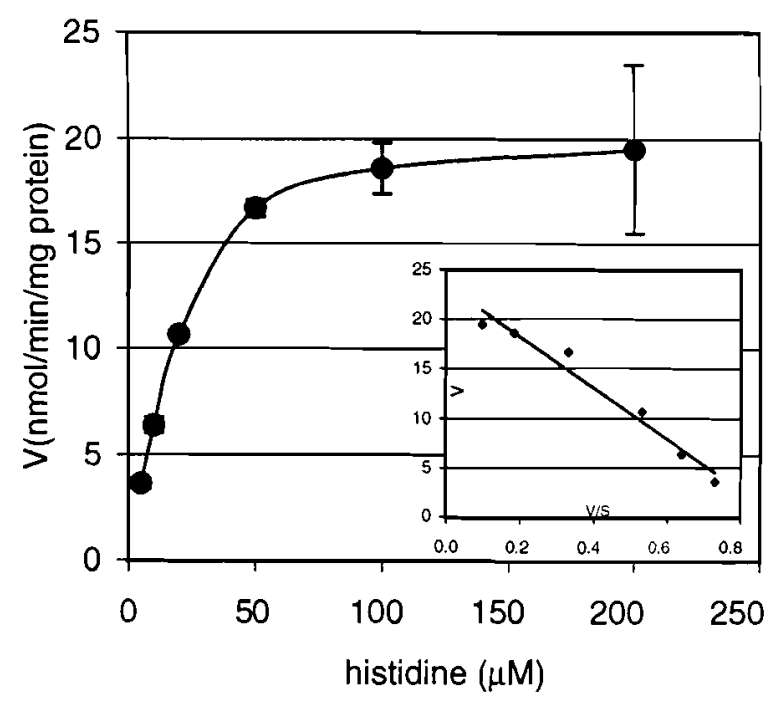

Fig. 6 Kinetics of ${ }^{14} \mathrm{C}$-labelled histidine uptake in the yeast strain JT 16 (PAAT1-YE). The insert shows an Eadie-Hofstee plot of the same data.

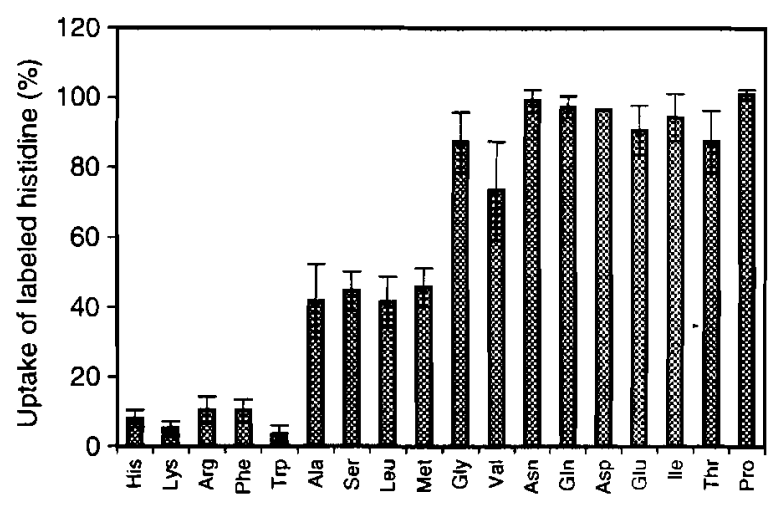

Fig. 7 Competition against AAT 1p-dependent ${ }^{14} \mathrm{C}$-labelled histidine uptake in yeast by non-labelled amino acids. The yeast strain JT16 (PAAT1-YE) was taken for uptake experiments, using a 10-told excess ( $2 \mathrm{~mm}$ ) of the competing amino acid.

To determine whether the $A A T 1$-encoded transporter has an affinity to amino acids other than histidine, competition studies were carried out. For this, a mixture of $0.2 \mathrm{~mm}$ of ${ }^{14} \mathrm{C}$-labelled histidine and $2 \mathrm{~mm}$ of an unlabelled amino acid were added to the cells and the apparent uptake rates were compared with those obtained with $0.2 \mathrm{~mm}{ }^{14} \mathrm{C}$-labelled histidine alone. As shown in Fig. 7, several amino acids showed significant competition against histidine uptake, in particular the positively charged amino acids lysine and arginine and the aromatic amino acids tryptophan and phenylalanine. A broad spectrum of amino acids was found to be moderately (alanine, serine, leucine, methionine, glycine, valine) competitive. Asparagine, glutamine, aspartate, glutamate, isoleucine, threonine and proline had no significant effect on histidine uptake.
However, the competitive ability of the amino acids does not necessarily mean that they are transported by AAT1p. To obtain further evidence for this, several yeast mutants defective in the uptake of various amino acids were used for complementation with AAT1 CDNA. We transformed the following mutants (in parentheses: uptake defect) with pAAT1-YE: HS100-3C (arginine: Sychrova and Chevallier, 1993); CD146 (methionine: Isnard et al., 1996); M4191, M4238 (valine, leucine, isoleucine: Didion et al., 1998); JGY51 (glutamine: Zhu et al., 1996); and 22( $\Delta$ )6AAL (lysine:W.-D. Fischer, unpublished data). In none of these strains, the presence of PAAT1-YE increased the uptake of ${ }^{14} \mathrm{C}$-labelled amino acids to levels above those of the control strains transformed with the vector pDR195. When the growth of amino acid uptake mutants was analysed on minimal media containing the amino acids in question, only one strain, $22(\Delta) 6 A A L$, was found to be complemented; while strain 22( $\triangle$ )6AAL (PDR195) required the dipeptide lysylleucine for growth on SC medium, growth on SC medium lacking lysylleucine was observed with $22(\triangle) 6 \mathrm{AAL}$ (PAAT1-YE) (data not shown).

\section{Transport properties of AAT1p in oocytes}

In order to confirm the transport activity and substrate specificity of AAT1p, as well as the suspected mechanism of proton symport, AAT1 CRNA-injected oocytes were used. For the assays, changes in the membrane potential were measured after the addition of individual amino acids at a concentration of $2 \mathrm{~mm}$, with the $\mathrm{pH}$ of the bathing solution adjusted to 5.0. In agreement with the complementation data obtained with yeast, histidine and lysine were found to be the best transport substrates (Fig. 8). In addition, several other amino acids were able to elicit membrane depolarization in $A A T 1$-injected oocytes. In most cases, amino acids that showed no or only weak competition of histidine uptake in yeast (aspartate, glutamate, isoleucine, threonine and proline) were also negative in membrane depolarization in oocytes. However, this correlation did not hold true for arginine and

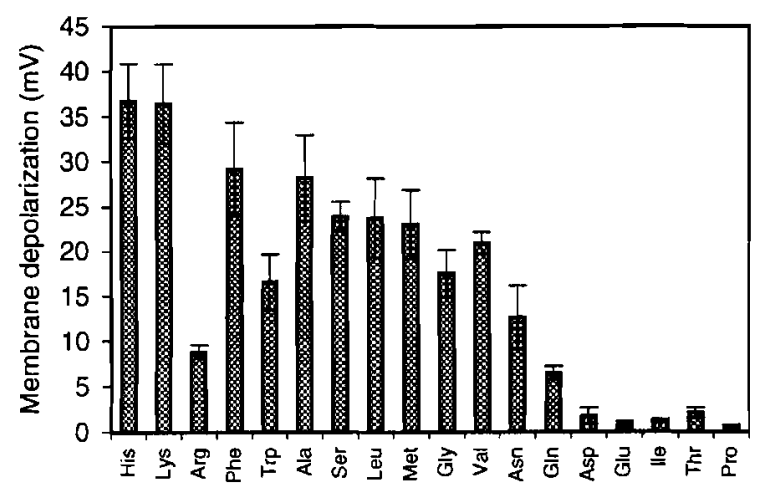

Fig. 8 Peak depolarizations of oocyte membranes induced by amino acids ( $2 \mathrm{~mm}$ ). Oocytes injected with AAT/ CRNA were used for the experiments. 


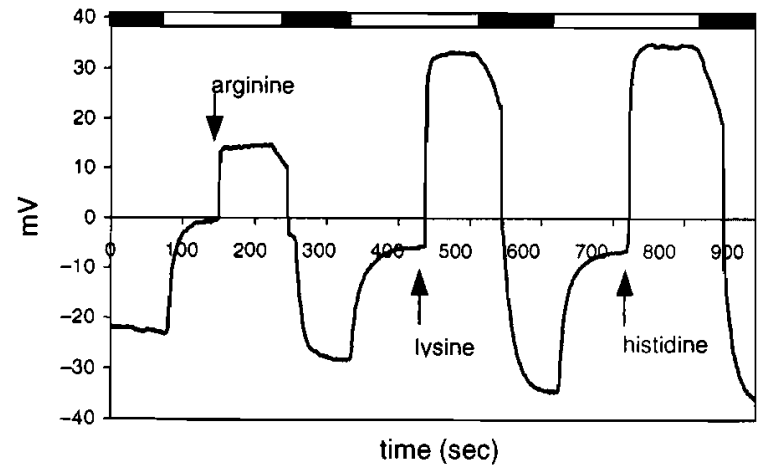

Fig. 9 Changes in membrane potential in a single, AAT expressing oocyte in response to the successive addition and removal of three different amino acids $(5 \mathrm{~mm}$ ) and to shifts in the $\mathrm{pH}$ of the bathing solution. ( $\square$ ) $\mathrm{pH} 7.5 ;(\square) \mathrm{pH} 5.0$

tryptophan, which are strong competitors in yeast but not effectively transported by AAT $1 p$ in oocytes.

In Fig. 9, a representative trace of amino acid-induced membrane potential changes in AATI CRNA-injected oocytes is shown. In a bathing solution at $\mathrm{pH} 5.0$, successive additions of arginine, lysine and histidine at concentrations of $5 \mathrm{~mm}$ each induced membrane depolarizations of 15.0, 39.1 and $41.1 \mathrm{mV}$, respectively. In contrast, no amino acid-induced membrane depolarization was observed in oocytes when the bathing solution was kept at pH 7.5 (data not shown). These results indicate a protondependent amino acid-induced net inward transfer of positive charge and are consistent with a proton symport mechanism.

\section{DISCUSSION}

Starting out from a haustorial cDNA library of Uromyces fabae, we have been able to isolate fungal genes that show a preferential expression in planta. Two of them were shown to be specifically expressed in haustoria, namely a putative amino acid transporter encoded by AAT2 (PIG2; Hahn et al., 1997) and a hexose transporter encoded by $H X T 1$ (Voegele et al., 2001). In the present paper we have characterized a gene encoding a second amino acid transporter, $A A T 1$. The AAT1-encoded protein exhibits $57 \%$ identity to AAT2p, more than to any other fungal amino acid transporter identified by BLAst search. Phylogenetically, AAT1p and AAT2p are closely related to the amino acid transporter AmAAP1 of Amanita muscaria (Nehls et al., 1999), the only published sequence of another basidiomycete amino acid transporter up to now.

$A A T 1$ and $A A T 2$ exhibit a very similar gene structure; not only do they both contain 17 introns, but 11 of these introns are at exactly the same positions within their coding regions. This observation clearly points towards $A A T 1$ and $A A T 2$ being the products of a gene duplication. Further evidence for this fact comes from heterologous Southern hybridization studies of genomic DNA from various fungi. While $A A T 1$ and $A A T 2$ CDNA probes did not hybridize to each other, they caused hybridization signals to two other rusts, but not to several other fungi. Based on these results we propose that the hypothetical gene duplication leading to $A A T 1$ and $A A T 2$ occurred in the course of evolution of the rust fungi, earlier than the splitting of the three rust species under analysis.

Despite their similar organization, the expression patterns of $A A T 1$ and $A A T 2$ are quite different. While both genes exhibit the highest transcript levels in haustoria, $A A T 1$ is already significantly expressed in germinating spores and throughout the development of the rust mycelium. During rust development, a gradual switch in the size of $A A T 1$ transcripts from $2.3 \mathrm{to} 2.5 \mathrm{~kb}$ was observed, with the smaller sized transcript dominating during the first few hours of infection structure formation. "Since genomic Southern hybridization indicated the presence of a single $A A T 1$ gene in the genome of $U$. fabae, and because $A A T I$ CDNA sequences obtained from germinated spores and from haustoria were found to be identical, the corresponding transcipts are probably from the same gene. It is possible that the size heterogeneity is due to the use of different initiation or termination sites during $A A T / 1$ transcription.

The transport properties of the $A A T 1$-encoded protein was investigated in two expression systems. In yeast, the histidine uptake deficiency of strain JT16 was complemented by the multicopy plasmid pAAT1-YE, allowing the transformants to grow on low histidine concentrations. Kinetic studies with ${ }^{14} \mathrm{C}$-histidine revealed AAT1p as a high-affinity histidine transporter. In addition, competition experiments demonstrated a high-affinity binding to AAT1p of basic and aromatic amino acids, and intermediate binding for several other amino acids. The expectation, however, that these amino acids are transported as well was only confirmed for lysine by complementation studies with the yeast mutant JT16. A reason for this could be an endogenous regulation of amino acid transport properties in yeast. Recently, Chen et al. (2001) observed that the Arabidopsis transporter ANT1 was able to complement the histidine uptake defect of yeast strain JT16 in the presence of $0.6 \mathrm{~mm}$, but not $6 \mathrm{~mm}$, arginine.

With Xenopus oocytes as the expression system, an often described advantage is a minimal activity of endogenous amino acid transport (Miller and Zhou, 2000). Regarding the amino acid transport of AAT1p, a similar but not identical picture as with yeast was observed; several other amino acids as well as histidine and lysine induced membrane depolarizations in AATI CRNAinjected cells. The only amino acids which were clearly negative in both expression systems were glutamate, aspartate, threonine, isoleucine and proline. Differences between the functional features of transporters expressed in oocytes, yeast or in planta have been reported (Dreyer et al., 1999; Tanner and Caspari, 1996). However, until now the only clue for an explanation was given by a mutational analysis of the plasma membrane water channel 
PM28A of Arabidopsis, which revealed different phosphorylation patterns when expressed in Xenopus oocytes or in planta (Johansson et al., 1998). The effects of protein phosphorylation might be on both targeting and substrate binding.

Depending on their lifestyles, fungi show different types of regulation of their nutrient uptake capabilities. For instance, the saprophytic yeast $S$. cerevisiae is well-adapted to a rapidly changing environment by a set of numerous amino acid transporters which are regulated by extracellular nutrients via specific sensor proteins (De Boer et al., 2000; Forsberg and Ljungdahl, 2001). In the ectomycorrhizal fungus Amanita muscaria, amino acid uptake from the soil is also regulated by nutrient levels. The low constitutive expression of the AmAAP1 gene encoding a broadspectrum amino acid transporter was found to be increased 10 fold in the absence of an appropriate nitrogen source (Nehls et al., 1999). In contrast, the biotrophic parasite U. fabae expresses nutrient transporters mainly in a developmentally controlled and cell-specific manner. For example, the putative amino acid transporter AAT2p and the hexose transporter HXT1p are only significantly expressed in the haustorial membrane, indicating that sugars and some amino acids are mainly taken up by haustoria (Hahn et al., 1997; Voegele et al., 2001). The absence of these transporters in intercellular hyphae might be explained by the low concentrations of sugars and amino acids in the apoplast compared to the symplast (Lohaus et al., 1995; Solomon and Oliver, 2001). Although metabolite concentrations in the extrahaustorial matrix are unknown, they are likely to be significantly higher than in the apoplast, and could provide haustoria with an increased nutrient supply. However, the expression levels of AAT1p in hyphae and haustoria, respectively, indicate that this transporter takes up amino acids both from the apoplast and the extrahaustorial matrix.

\section{EXPERIMENTAL PROCEDURES}

\section{Organisms}

Yeast strains were grown on SC medium containing the appropriate supplements (Sherman et al., 1986) S. cerevisiae strain JT16 (Tanaka and Fink, 1985) was obtained from G. Fink. Strain 22 6 AAL (put4, uga4, gap1, can1, lyp1, alp1, lys2), a derivative of 22574d (Jauniaux et al., 1987), was obtained from W.N. Fischer and W. Frommer (Tübingen, Germany). Due to the lys 2 and the lypl and alp1 mutations, 22 $\triangle 6 \mathrm{AAL}$ is lysine auxotrophic and unable to take up lysine; therefore, it does not grow on SC medium unless lysine is provided as a dipeptide, such as lysylleucine (W.N. Fischer, personal communication).

The cultivation of broad bean plants (Vicia fabacv. con Amore), their inoculation with Uromyces fabae race $I_{2}$ uredospores, and the isolation of rust haustoria was performed as described by Hahn and Mendgen (1997). In vitro infection structures of
$U$. fabae were generated on scratched polyethylene sheets as described by Deising et al. (1991). The propagation of Uromyces vignae and Puccinia graminis was carried out as previously described (Hahn and Mendgen, 1992).

\section{Nucleic acid manipulation}

Isolation of fungal RNA and DNA was performed as described (Hahn and Mendgen, 1997). Screening of the genomic EMBL3 library from Uromyces fabae (Hahn and Mendgen, 1992) and other nucleic acid manipulations were done using standard molecular biology techniques.

Genomic Southern hybridizations using digoxigenin-labelled probes were performed according to Engler-Blum et al. (1993), at a hybridization temperature of $60^{\circ} \mathrm{C}$. The $A A T 7$ CDNA probe consisted of a mixture of two PCR fragments covering the complete coding region (bases 1009-4287 of the genomic AAT1 sequence (AJ308252) excluding introns); the AAT2 CDNA probe consisted of a mixture of two PCR fragments covering codons 1-527 (bases 1806-4898 of the genomic AAT2 sequence (U81794) excluding introns).

\section{DNA sequencing and phylogenetic analysis}

Sequencing was performed with dye-labelled terminators using a sequencing kit and an automatic sequencer (Applied Biosystems, Foster City, CA). Homology searches were done using the BLAST algorithm (Altschul et al., 1990). Multiple sequence alignments of AAT1p with other amino acid transporters were generated using the program CLUSTAL W (1.8) (Thompson et al., 1997).

\section{Expression studies in yeast}

For the functional expression of $A A T 1$, a cDNA carrying the $A A T 1$ coding region was amplified. For expression in yeast, a 5' primer (TCATGGATCAAGAATTCGTAACTG) covering an ATG located $57 \mathrm{bp}$ (corresponding to 19 codons) downstream of the putative ATG start codon, and a $3^{\prime}$-primer (TCCGCTCATGAAAACCTC) covering a region downstream of the TAG stop codon, were chosen for RT-PCR, using haustorial mRNA of $U$. fabae as template. With these primers, a 1781 bp AAT1 CDNA fragment was amplified using Pfu polymerase (Stratagene, La Jolla, CA) and cloned into the $X$ hol site (filled in with Klenow enzyme) of the yeast expression vector pDR195 (Rentsch et al., 1995). The resulting PAAT1-YE plasmid was used for the transformation of Saccharomyces cerevisiae strains defective in the uptake of amino acids. Transformation of yeast was carried out as described by Dohmen et al. (1991).

S. cerevisiae strain JT16 (pAAT1-YE) was used for uptake studies with ${ }^{14} \mathrm{C}$-labelled histidine as a substrate. Kinetic analyses were performed similarly to the protocol described by (Fischer et al., 1995). Transformed yeast cells were grown in $0.67 \%$ yeast 
nitrogen base without amino acids (YNB: Sherman et al., 1986) containing $2 \%$ glucose and $6 \mathrm{~mm}$ histidine, to an $\mathrm{OD}_{600}$ of 0.5 . Cells were pelleted, washed once with water and resuspended in $0.67 \%$ YNB containing $2 \%$ glucose to an $0_{600}$ of 12 . Before starting the transport assays $100 \mu \mathrm{L}$ of cell suspension were diluted 1 : 5 with YNB and equilibrated for $5 \mathrm{~min}$ at $30{ }^{\circ} \mathrm{C} .{ }^{14} \mathrm{C}$-labelled histidine $(5-100 \mu \mathrm{m}, 1-2 \mathrm{Ci} / \mathrm{mol})$ was added and aliquots of $150 \mu \mathrm{L}$ were taken after 60,120 and $240 \mathrm{~s}$, placed on to glass fibre filters and immediately washed with $8 \mathrm{~mL}$ of ice-cold water. Filters were transferred to scintillation liquid and incubated for $1 \mathrm{~h}$ at room temperature in darkness. The radioactivity was measured in a LS1801 scintillation counter (Beckman). All experiments were done in triplicate. The data presented are the means of one representative experiment out of three independent experiments.

\section{Expression studies in Xenopus oocytes}

For expression in Xenopus oocytes the vector pBF1 (W. Frommer, Tübingen, Germany) was used, which confers expression from an SP6 RNA polymerase promoter and adds a poly $(A)_{80}$ tail to the ends of the in vitro generated transcripts. The AAT1 CDNA was cloned into pBF1 (cut with $\mathrm{NcOl}$ and $X$ hol) as a 1837 bp RT-PCR fragment containing overhangs (underlined) compatible with $\mathrm{NCOl}$ on the 5' primer (CATGAGCTCGGACGATGTGAAGG), and compatible with $X$ hol on the $3^{\prime}$ primer (TCGAAGTACAGCCTA(AGCG(AAG), respectively. The overhangs were generated by amplification of two PCR fragments that overlapped at their ends by the underlined bases, followed by heat-denaturation and subsequent annealing of a mixture of these fragments. In the resulting plasmid, the cloned cDNA contained the putative start codon of $A A T 1$ within the $N$ col site. The AATI CRNA was transcribed in vitro using SP6 RNA polymerase (MessageMachine, Ambion, Austin, TX), after linearization of the plasmid with Mlul, which cuts immediately after the poly(A) tail.

Xenopus oocytes were prepared as described (Voegele et al., 2001). Oocytes were injected with $50 \mathrm{ng}$ AAT1 CRNA (1 ng/nL). Measurements of oocyte membrane potentials were carried out 5-6 days after CRNA injection in transport buffer containing $96 \mathrm{~mm}$ choline chloride, $2 \mathrm{mM} \mathrm{CaCl}, 10 \mathrm{~mm} \mathrm{MES}$, with a pH varying between 7.5 and 5.0. Output voltages were recorded using microelectrodes filled with $3 \mathrm{M} \mathrm{KCl}$ and a resistance of approximately 7$9 \mathrm{M} \Omega$, using a VF-180 amplifier (Bio-Logic, Echirolles, France).

\section{ACKNOWLEDGEMENTS}

This work was carried out in the laboratory of Kurt Mendgen to whom we are indebted for continuous support. We thank Uwe Ludewig and Wolf Frommer (Tübingen) for support in establishing the oocyte system; Nataliya Kucheryava for help with plasmid constructions; Dana Zejda for assistance in Xenopus surgery; and Ralf Voegele for critically reading of the manuscript. This research was supported by grants from the Deutsche Forschungsgemeinschaft (Me 523/23-1 and Ha 1486/2-1).

\section{REFERENCES}

Altschul, S.F., Gish, W., Miller, W., Myers, E.W. and Lipman, D. (1990) Basic local alignment search tool. J. Mol. Biol. 215, 403-410.

Chen, L., Ortiz-Lopez, A., Jung, A. and Bush, D.R. (2001) ANT1, an aromatic and neutral amino acid transporter in Arabidopsis. Plant Physiol. 125, 1813-1820.

De Boer, M., Nielsen, P.S., Bebelman, J.-P., van Heerikhuizen, H., Andersen, H.A, and Planta, R.J. (2000) Stp1p, Stp2p and Abf1p are involved in regulation of expression of the amino acid transporter gene BAP3 of Saccharomyces cerevisiae. Nucl. Acids Res. 88, 974981.

Deising, H., Jungblut, P.R. and Mendgen, K. (1991) Differentiationrelated proteins of the broad bean rust fungus Uromyces viciae-fabae, as revealed by high resolution two-dimensional polyacrylamide gel electrophoresis. Arch. Microbiol. 155, 191-198.

Didion, T., Regenberg, B., Jorgensen, M.U., Kielland-Brandt, M.C. and Andersen, H.A. (1998) The permease homologue Ssy1p controls the expression of amino acid and peptide transporter genes in Saccharomyces cerevisiae. Mol. Microbiol. 27, 643-650.

Dohmen, R.J., Strasser, A.W., Honer, C.B. and Hollenberg, C.P. (1991) An efficient transformation procedure enabling long-term storage of competent cells of various yeast genera. Yeast, 7, 691-692.

Dreyer, I., Horeau, C., Lemaillet, G., Zimmermann, S., Bush, D.R., Rodriguez-Navarro, A., Schachtman, D.P., Spalding, E.P., Sentenac, H. and Gaber, R.F. (1999) Identification and characterization of plant transporters using heterologous expression systems. J. Exp. Bot. 50, 1073-1087.

Engler-Blum, G., Meier, M., Frank, J. and Müller, G.A. (1993) Reduction of background problems in nonradioactive Northern and Southern analyses enables higher sensitivity than ${ }^{32} \mathrm{P}$-based hybridizations. Anal. Biochem. 210, 235-244.

Fischer, W.-N., Kwart, M., Hummel, S. and Frommer, W.B. (1995) Substrate specificity and expression profile of amino acid transporters (AAPs) in Arabidopsis. J. Biol. Chem. 270, 16315-16320.

Forsberg, H. and Ljungdahl, P.O. (2001) Genetic and biochemical analysis of the yeast plasma membrane Ssy1p-Ptr3p-Ssy5p sensor of extracellular amino acids. Mol. Cell Biol. 21, 814-826.

Hahn, M. (2000) The Rust Fungi. Cytology, Physiology and Molecular Biology of Infection. In: Fungal Pathology. (Kronstad, J.W., ed.) Dordrecht: Kluwer Academic Publishers, pp. 267-306.

Hahn, M. and Mendgen, K. (1992) Isolation by ConA binding of haustoria from different rust fungi and comparison of their surface qualities. Protoplasma, 170, 95-103.

Hahn, M. and Mendgen, K. (1997) Characterization of in planta-induced rust genes isolated from a haustorium-specific CDNA library. Mol. PlantMicrobe interact. 10, 427-437.

Hahn, M., Neef, U., Struck, C., Göttfert, M. and Mendgen, K. (1997) A putative amino-acid transporter is specifically expressed in haustoria of the rust fungus Uromyces fabae. Mol. Plant-Microbe interact. 10 , 438-445.

Isnard, A.-D., Thomas, D. and Surdin-Kerjan, Y. (1996) The study of methionine uptake in Saccharomyces cerevisiae reveals a new family of amino acid permeases. J. Mol. Biol. 262, 473-484. 
Jack, D.L., Paulsen, I.T. and Saier, M.H. (2000) The amino acid/ polyamine/ organocation (APC) superfamily of transporters specific for amino acids, polyamines and organocations. Microbiology, 146, 1797-1814

Jäger, K. and Reisener, H.-J. (1969) Host-parasite metabolic relationship between Puccinia graminis var. tritici and Triticum aestivum (wheat). I. Uptake of amino acids from the host. Planta, 85, 57-72.

Jauniaux, J.-C., Vandenbol, M., Vissers, S., Broman, K. and Grenson, M. (1987) Nitrogen catabolite regulation of proline permease in Saccharomyces cerevisiae. Cloning of the PUT4 gene and study of PUT4 RNA levels in wild-type and mutant strains. Eur. J. Biochem. 164,601606.

Johansson, I., Karlsson, M., Shukla, V.K., Chrispeels, M.J., Larsson, C. and Kjellbom, P. (1998) Water transport activity of the plasma membrane aquaporin PM28A is regulated by phosphorylation. Plant Cell, 10, $451-459$.

Lohaus, G., Winter, H., Riens, B. and Heldt, H.W. (1995) Further studies of the phloem loading process in leaves of barley and spinach. The comparison of metabolite concentrations in the apoplastic compartment with those in the cytosolic compartment and in the sieve tubes. Bot Acta, 108, 270-275.

Mendgen, K. (1981) Nutrient uptake in rust fungi. Phytopathology, 71 983-989.

Mendgen, K., Struck, C., Voegele, R.T. and Hahn, M. (2000) Biotrophy and rust haustoria. Physiol. Mol. Plant Pathol. 56, $141-145$

Miller, A.J. and Zhou, J.J. (2000) Xenopus 0ocytes as an expression system for plant transporters. Biochim. Biophys. Acta, 1465, 343-358.

Nehls, U., Kleber, R., Wiese, J. and Hampp, R. (1999) Isolation and characterization of a general amino acid permease from the ectomycorrhizal fungus Amanita muscaria. New Phytol. 144, 343-349.

Rentsch, D., Laloi, M., Rouhara, I., Schmelzer, E., Delrot, S. and Frommer, W. (1995) NTR1 encodes a high affinity oligopeptide transporter in Arabidopsis. FEBS Lett. 370, 264-268.
Sherman, F., Fink, G.R. and Hicks, J.B. (1986) Methods in Yeast Genetics. New York: Cold Spring Harbor Laboratory.

Solomon, P.S. and Oliver, R.P. (2001) The nitrogen content of the tomato leaf apoplast increases during infection by Cladosporium fulvum. Planta, 213, 241-249.

Staples, R.C. (2000) Research on the rust fungi during the twentieth century. Ann. Rev. Plant Pathol. 38, 49-69.

Struck, C., Hahn, M. and Mendgen, K. (1996) Plasma membrane $\mathrm{H}^{+}$ ATPase activity in spores, germ tubes and haustoria of the rust fungus Uromyces viciae-fabae. Fungal Genet. Biol. 20, 30-35.

Struck, C., Siebels, C., Rommel, O., Wernitz, M. and Hahn, M. (1998) The plasma membrane $\mathrm{H}^{+}$-ATPase from the biotrophic rust fungus Uromyces fabae: Molecular characterization of the gene (PMA 1 ) and functional expression of the enzyme in yeast. Mol. Plant-Microbe Interact. $11,458-465$

Sychrova, H. and Chevallier, M.R. (1993) Cloning and sequencing of the Saccharomyces cerevisiae gene $L Y P 1$ coding for a lysine-specific permease. Yeast, 9, 771-782.

Tanaka, J, and Fink, G.R. (1985) The histidine gene (HIP1) of Saccharomyces cerevisiae. Gene, 38, 205-214.

Tanner, W. and Caspari, T. (1996) Membrane transport carriers. Ann. Rev. Plant Physiol. Plant Mol. Biol. 47, 595-626.

Thompson, J.D., Gibson, T.J., Plewniak, F., Jeanmougin, F. and Higgins, D.G. (1997) The ClustalX windows interface: flexible strategies for multiple sequence alignment aided by quality analysis tools. Nud. Acids Res. 24, 4876-4882.

Voegele, R.T., Hahn, M., Struck, C. and Mendgen, K. (2001) The role of rust haustoria in sugar supply during infection of broad bean by the rust fungus Uromyces fabae. Proc. Nat/ Acad. Sci. USA, 98, 8133-8138.

Zhu, X., Garrett, J., Schreve, J. and Michaeli, T. (1996) GPN1, the highaffinity glutamine permease of Saccharomyces cerevisiae. Curr. Genet. $30,107-114$ 